.
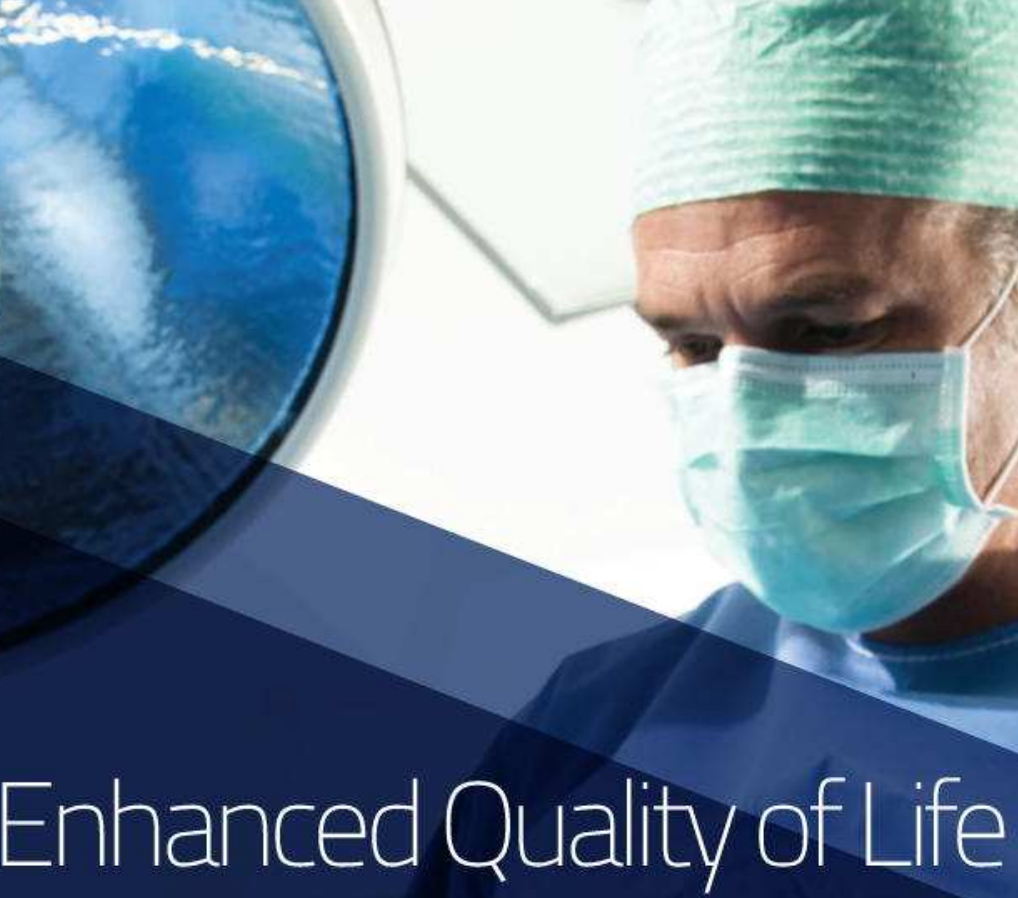

8
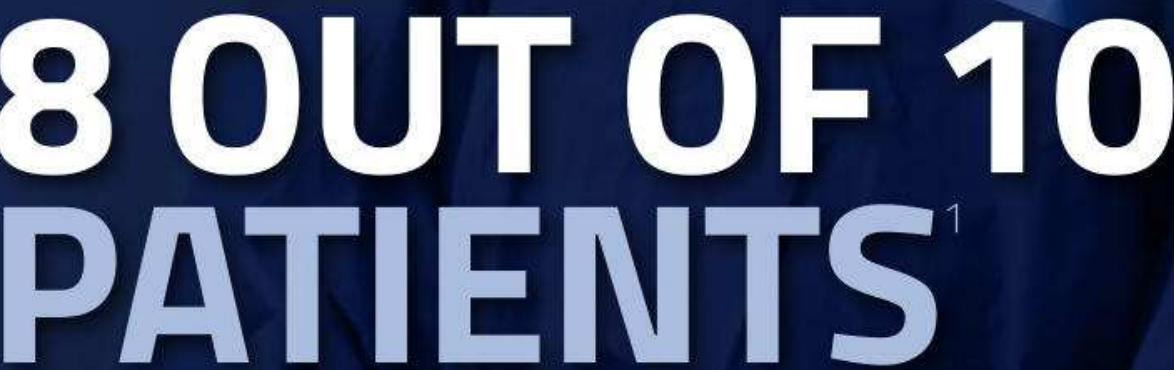

Experienced Improved Ejection Fraction ${ }^{2}$ or NYHA Heart Failure Class ${ }^{2}$ After a Protected PCI procedure with the Impella ${ }^{\circledR}$ Heart Pump

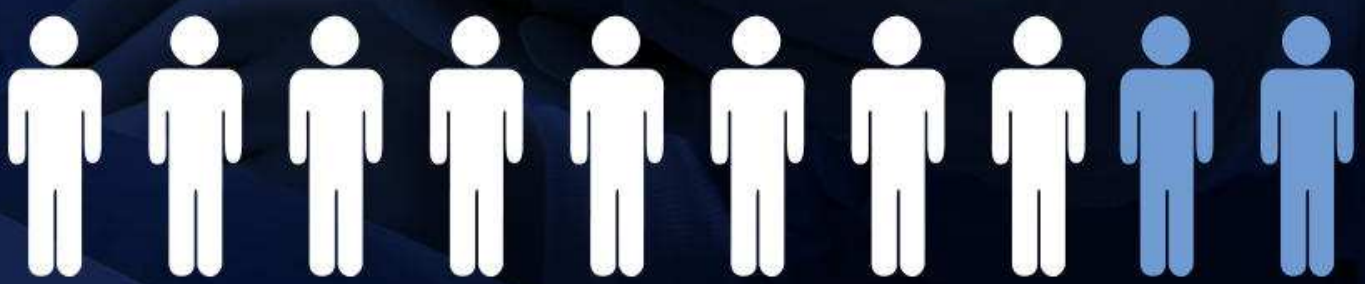

Learn more about Impella and how to build a Protected PCI program visit www.protectedpci.com 


\title{
Randomized Clinical Trial on Short-Time Compression with Kaolin-Filled Pad: A New Strategy to Avoid Early Bleeding and Subacute Radial Artery Occlusion after Percutaneous Coronary Intervention
}

\author{
LUIGI POLITI, M.D., ALESSANDRO APRILE, M.D., CATIA PAGANELLI, M.D., ANDREA AMATO, M.D., \\ GIUSEPPE B. ZOCCAI, M.D., FABIO SGURA, M.D., DANIEL MONOPOLI, M.D., ROSARIO ROSSI, M.D., \\ MARIA G. MODENA, M.D., and GIUSEPPE M. SANGIORGI, M.D.
}

From the Interventional Cardiology, Policlinico Hospital, University of Modena and Reggio Emilia, Modena, Italy

\begin{abstract}
Background: Despite the increasing use of transradial techniques for cardiac percutaneous procedures, none of the strategies commonly utilized for hemostasis has been able to reduce the occurrence of radial artery occlusion (RAO). The aim of this study was to evaluate the occurrence of 24-hour RAO and the rate of bleeding of a novel hemostatic device for radial closure after percutaneous interventions, in adjunct to short-time compression. Methods: Once the radial access was obtained, patients were randomized to 3 different strategies of radial closure: a short compression with the QuikClot ${ }^{\circledR}$ Interventional ${ }^{\mathrm{TM}}$ pad (Z-Medica Corporation, Wallingford, CT, USA) (15 minutes, group 1), a short compression (15 minutes, group 2), and a conventional prolonged compression (2 hours, group 3) both without QuikClot ${ }^{\circledR}$ utilization.

Results: Fifty patients in group 1, 20 in group 2, and 50 in group 3 were enrolled. The three groups were homogenous for baseline and procedural characteristics. None of patients in group 1 developed RAO, $1(5 \%)$ occurred in group 2 , and $5(10 \%)$ in group $3(P=0.05)$. Active bleeding after compression removal occurred in 10 patients $(20 \%)$ in group $1,18(90 \%)$ in group 2 , and $1(2 \%)$ in group $3(P<0.001)$. Among patients in group 1 , at univariate analysis, the predictors of acute bleeding resulted in chronic therapy with clopidogrel (Odds Ratio 28.78, 95\% Confidence Intervals 4.79-172.82, P $<0.001)$ and high levels of activated clotting time (ACT) at the time of sheath removal (OR 1.02, 95\% CI 1.00-1.03, $P=0.009)$. At ROC analysis, the cutoff value of ACT for the risk of bleeding with a sensitivity of $80 \%$ and specificity of $75 \%$ was 287 seconds.

Conclusions: Early sheet removal and short-time compression with QuikClot ${ }^{\circledR}$ Interventional ${ }^{\mathrm{TM}}$ can reduce the rate of RAO after diagnostic or interventional procedures especially in patients not on double antiplatelet therapy. (J Interven Cardiol 2011;24:65-72)
\end{abstract}

\section{Introduction}

Transradial access for percutaneous cardiovascular procedures provides a low rate of local complications and similar clinical results compared to other sites of access. ${ }^{1-3}$ Sheath removal after transradial catheterization is conventionally based on external compression

Address for reprints: Giuseppe M. Sangiorgi, M.D., Interventional Cardiology, Policlinico Hospital, University of Modena and Reggio Emilia, Via del Pozzo 71, Modena 41100, Italy. Fax: 39-059-4224496; e-mail: gsangiorgi@gmail.com achieved with a simple bandage applied to the entry site. ${ }^{4}$ Observational studies have shown that prolonged or aggressive compression techniques favor local complications such as radial artery occlusion (RAO). ${ }^{4,5}$ RAO is relatively infrequent $(5 \%-10 \%$ of cases $)^{6,7}$ and usually considered as a quiescent complication of transradial procedures because of the dual circulation of the palmar arch. Yet, its occurrence precludes future transradial access forcing the shift to the femoral access, which is responsible for more bleeding complications and prolonged hospitalization as well as greater discomfort for the patient. Therefore, 
it seems reasonable to preserve radial patency by keeping compression time as short as possible. Dedicated hemostatic devices have been developed over the past few years, ${ }^{5,8,9}$ but the advantages in terms of efficacy of these tools compared to conventional manual compression technique have never been demonstrated. QuikClot $^{\circledR}$ Interventional ${ }^{\mathrm{TM}}$ bandage (Z-Medica Corporation, Wallingford, CT, USA) is a novel hemostatic device, consisting of a pro-coagulant (Kaolin) agent-filled hydrophilic nonwoven pad that can be applied topically as an adjunct to manual compression and is indicated for the local management and control of surface bleeding from vascular access sites. ${ }^{10,11}$ When Kaolin comes into contact with blood in a wound, it rapidly absorbs the smaller water molecules from the blood. The larger platelets and clotting factor molecules remain in the wound in a highly concentrated form promoting a rapid natural clotting. The aim of this prospective three-arm randomized study was to evaluate the safety and efficacy of QuikClot ${ }^{\mathrm{Q}}$ in adjunct to short-time compression, compared to either short- or prolonged-time compression techniques with conventional sterile gauzes after transradial diagnostic or interventional coronary procedures.

\section{Methods}

All consecutive patients undergoing transradial elective diagnostic or interventional coronary procedures between November 1, 2009 and January 31, 2010 at the Catheterization Laboratory of Modena University Hospital were considered to be enrolled in the study. The only exclusion criteria were an abnormal Allen's test before puncture and failure to provide written informed consent. Once the radial access was obtained, patients were randomized into 3 arms corresponding to 3 different strategies of radial closure. Randomization was based on a computer-generated randomization list. Clinical variables were obtained by review of hospital charts and patient interview; any medication that could potentially affect bleeding was noted, and systolic and diastolic blood pressure were measured at the end of the procedure. Patients receiving chronic warfarin therapy before hospital admission were noted; in all cases, warfarin was stopped at least 3 days before the procedure until International Normalized Ratio (INR) was $<1.5$ and low molecular weight heparin (LMWH) was administered. The study protocol was approved by the institutional investigation committees and all patients signed informed consent.
Radial Access. After sterile preparation and injection of $2 \%$ lidocaine at the puncture site, a 20 -gauge needle was used to enter the radial artery $5 \mathrm{~cm}$ above the crease of the wrist using Seldinger's technique. On the appearance of pulsatile flow, a Terumo ${ }^{\circledR}$ (Terumo Corporation, Tokyo, Japan) 0.018-inches guidewire was advanced into the radial artery lumen. A 6-Fr glide sheath was then advanced over the guidewire into the radial artery (Radifocus ${ }^{\circledR}$ Introducer II, Terumo Corporation, Tokyo, Japan). Two hundred micrograms of nitroglycerin or $5 \mathrm{mg}$ of diltiazem were administered diluted in a 20 - $\mathrm{mL}$ syringe intraarterially. In addition, a standard dose of 5,000 IU of unfractionated heparin was administered, followed by additional doses in case of coronary intervention to achieve and maintain activated clotting time $($ ACT $)>250$ seconds. The ACT was measured in all patients at the end of the procedure from the arterial introducer sheath using the Hemochron ${ }^{\circledR}$ (ITC, Edison, NJ, USA) system. All arterial sheaths were removed immediately after the diagnostic or interventional procedure irrespectively of the ACT value.

Randomization to Closure Techniques. After achieving the radial access and based on a computergenerated randomization list, patients were allocated into one of the following strategy groups. Group 1: the compression of radial artery was obtained with QuikClot ${ }^{\circledR}$ Interventional ${ }^{\mathrm{TM}}$ hemostatic pad applied directly on the skin with a folded gauze over the pad. All the dressing was then wrapped with adhesive tape and maintained for 15 minutes. After this period, the compression was removed leaving the QuikClot ${ }^{\circledR}$ pad for 2 hours secured with a Tegaderm ${ }^{\mathrm{TM}}$ (3M Company, St. Paul, MN, USA) adhesive bandage (Fig. 5). Group 2: hemostasis was obtained by direct compression of the radial artery with a folded conventional sterile gauze wrapped with tape and maintained for 15 minutes only (control group, similar to group 1 without the tested pad). After this period, the compression was removed leaving a sterile gauze secured with a Tegaderm ${ }^{\mathrm{TM}}$ adhesive bandage. Group 3: standard technique consisting of direct compression of the site of puncture with a folded conventional sterile gauze wrapped with tape and maintained for 2 hours (conventional strategy).

End-points of the Study. The main end-point was subacute RAO; the secondary end-point was failure of the closure technique. Death, myocardial infarction, or major bleeding occurring in hospital was appraised as a secondary end-point. 


\section{SHORT-TIME RADIAL COMPRESSION WITH KAOLIN-FILLED PAD}

Radial Artery Occlusion. Radial artery patency was assessed using the Barbeau's test ${ }^{12}$ at 24 hours after the procedure. The investigator assessing the artery patency was blinded to the closure technique used. A pulse oximeter sensor was placed over the index fingertip to obtain a plethysmographic signal. Both radial and ulnar arteries were compressed to observe a loss of the plethysmographic signal. Next, the radial artery was released, and the return of the plethysmographic signal was observed. A return of the signal confirmed radial artery flow and, hence, patency. An absence of the return of the signal was interpreted as RAO. The ulnar artery was then released to observe the return of the signal, confirming proper functioning of the equipment. All findings of occlusion were confirmed ultrasonographically by another investigator. Efficacy was defined as a radial artery patency confirmed by the presence of plethysmographic signal and ultrasonographic wave 24 hours after the procedure.

Closure Technique Failure. After the scheduled time of compression ( 15 minutes for groups 1 and 2 and 2 hours for group 3 ), the entry site was revised for signs of active bleeding (acknowledged as failure of the closure strategy). In case of failure, the compression was restored using the conventional strategy that was maintained for additional 2 hours and observed thereafter until bleeding stop. Safety was defined as absence of bleeding from the radial access site after the scheduled time from sheath removal in the different groups.

Statistical Analysis. Data are expressed as percentages or mean \pm standard deviation. Analysis was done by intention-to-treat. Comparison across groups were performed using Analysis of Variance (ANOVA) test for continuous variables and Pearson's chi-square test or Fisher's exact test where appropriate for categorical variables. Receiver operator characteristics (ROCs) analysis was used to test the value of ACT associated with greater sensitivity and specificity in predicting failure of closure technique. Logistic regression analysis was used to identify univariate predictors of outcomes. All tests were two-tailed and $\mathrm{P}<0.05$ was used for statistical significance. The Statistical Package for Social Sciences, version 15.0, software (SPSS, Chicago, IL, USA) was used for analysis.

\section{Results}

Clinical and Procedural Data. Of the planned 150 patients (50 for each group), 120 patients were en- rolled in the study and followed for end-points- 50 in group 1, 20 in group 2, and 50 in group 3. For ethical reasons, the enrollment in group 2 was stopped after the 20th patient because of high rate of active bleeding after compression removal (18 out of 20 patients). Subsequently, the randomization was continued only for group 1 and 3 until the achievement of 50 patients per group. Mean age was $61.8 \pm 13.2$ years, $72.5 \%$ of the patients were male and $15 \%$ were diabetics. Interventional procedures were performed in $59.2 \%$ of cases. Right radial artery was used in $86.7 \%$ of cases, left radial artery in the remainder. The three groups were homogenous for baseline and procedural characteristics (Tables 1 and 2). No major adverse events such as death, myocardial infarction, or major bleeding occurred during the hospitalization in any of the randomization groups.

Radial Artery Occlusion. None of the patients enrolled in group 1 developed the main outcome variable. Among patients enrolled in group 2 RAO occurred in 1 case (5\%) and among group 3 in 5 cases $(10 \%)(\mathrm{P}=0.05$, Fig. 1). The predictors of RAO in group 3 were older age (OR 1.09, 95\% CI 1.00-1.18, $\mathrm{P}=0.049)$ and smaller amount of intraprocedural heparin infused (OR 0.70, 95\% CI 0.49-0.99, $\mathrm{P}=0.044$ ) (Table 3), while no predictors could be appraised in group 2.

Failure of Closure Device. Active bleeding after compression removal occurred in 10 patients (20\%) in group 1, $18(90 \%)$ in group 2, and $1(2 \%)$ in group 3 ( $\mathrm{P}<0.001$, Fig. 2). In all cases, hemostasis was achieved with a supplementary compression for 2 hours that did not produced any RAO in group 1 . Among patients enrolled in group 1, at univariate analysis, the only predictors of acute bleeding resulted chronic therapy with clopidogrel (OR 28.78, 95\% CI 4.79-172.82, $\mathrm{P}<0.001)$ and high levels of ACT at the time of sheath removal (OR 1.02, 95\% CI 1.00-1.03, $\mathrm{P}=0.009$, Table 4 ). Among group 2 and group 3, none of the baseline clinical or procedural variables were associated with a significantly increased risk of active bleeding. At ROC analysis, when ACT was below 200 seconds none of the patients developed bleeding in group 1, while all patients with a value greater than 399 developed it. The cutoff value with a sensitivity of $80 \%$ and specificity of $75 \%$ was 287 seconds. The area under the curve was 0.761 ( $\mathrm{P}<0.001$, Fig. 3).

Clinical Follow-Up. A telephone contact at 6 months was initiated to all 6 patients ( 1 in group 2 and 5 in group 3) who have experienced RAO. They were 
POLITI, ET AL.

Table 1. Baseline Characteristics

\begin{tabular}{lcccc}
\hline Variable & Group $1 \mathrm{~N}=50$ & Group 2 N=20 & Group 3 N = 50 & P Value \\
\hline Age (years) & $64.16 \pm 11.53$ & $61.30 \pm 14.22$ & $59.72 \pm 14.23$ & 0.241 \\
Male sex & $37(74 \%)$ & $14(70 \%)$ & $36(72 \%)$ & 0.939 \\
BSA $\left(\mathrm{m}^{2}\right)$ & $1.87 \pm 0.15$ & $1.90 \pm 0.18$ & $1.93 \pm 0.17$ & 0.279 \\
Height $(\mathrm{cm})$ & $171.60 \pm 8.29$ & $171.20 \pm 6.76$ & $171.38 \pm 6.69$ & 0.977 \\
Weight $(\mathrm{kg})$ & $76.42 \pm 11.13$ & $80.00 \pm 14.96$ & $82.02 \pm 13.26$ & 0.091 \\
Diabetes mellitus & $6(12 \%)$ & $4(20 \%)$ & $8(16 \%)$ & 0.676 \\
Hypertension & $29(58 \%)$ & $13(65 \%)$ & $32(64 \%)$ & 0.781 \\
Dyslipidemia & $29(58 \%)$ & $11(55 \%)$ & $33(66 \%)$ & 0.602 \\
Previous MI & $14(28 \%)$ & $4(20 \%)$ & $12(24 \%)$ & 0.766 \\
Previous radial puncture & $3(6 \%)$ & $2(10 \%)$ & $4(8 \%)$ & 0.835 \\
Chronic aspirin therapy & $46(92 \%)$ & $19(95 \%)$ & $50(100 \%)$ & 0.132 \\
Chronic clopidogrel therapy & $10(20 \%)$ & $2(10 \%)$ & $11(22 \%)$ & 0.505 \\
LMWH administration & $8(16 \%)$ & $5(25 \%)$ & $5(10 \%)$ & 0.274 \\
Warfarin before hospital admission & $6(12 \%)$ & $3(15 \%)$ & $3(6 \%)$ & 0.435 \\
\hline
\end{tabular}

$\mathrm{BSA}=$ body surface area; $\mathrm{MI}=$ myocardial infarction; $\mathrm{LMWH}=$ low molecular weight heparin.

all alive, no major cardiac events occurred, and they did not notice any discomfort at the arm corresponding to the site of puncture.

\section{Discussion}

Despite the widespread and increasing use of transradial techniques for cardiac and peripheral percutaneous procedures, none of the strategy or devices commonly used for hemostasis have been able to avoid the occurrence of RAO, likely because these methods need prolonged artery compression. In the present study, we utilized a new compression strategy in order to reduce the incidence of subacute RAO consisting in applying the QuikClot ${ }^{\circledR}$ pad on the radial access with a compression maintained for only 15 minutes, leaving thereafter only the dressing with a simple bandage. This trial tested the occurrence of 24-hour RAO and the rate of bleeding after compression removal comparing a short compression with the QuikClot ${ }^{\circledR}$ Interventional ${ }^{\mathrm{TM}}$ pad versus two other strategies, a short compression and a conventional prolonged compression both without QuikClot ${ }^{\circledR}$ utilization. The main result of the trial is that QuikClot ${ }^{\circledR}$ utilization prevents RAO $(0$ cases compared to $5 \%$ and $10 \%$, respectively, in group 2 and 3 , $\mathrm{P}=0.05)$ with a relatively low risk of access site bleeding after its removal (bleeding occurred in 20\% of cases in group 1 compared to $90 \%$ in group 2 and $2 \%$ in group $3, \mathrm{P}<0.001)$. The occurrence of subacute RAO in the conventional group is similar to that reported in the literature ${ }^{6,7}$ and much higher than in the other two groups. This finding underscores the importance of a short-duration compression to reduce this complication. The reason of higher rate of RAO in patients of group 3 than those of group 1 with long compression after bleeding may be simply related to higher number of cases (50 vs. 10). Moreover, we assume also that the higher levels of ACT and the more intense antiplatelets regimen in the 10 patients enrolled in group 1 may have reduced the risk of RAO. Although

Table 2. Procedural Characteristics

\begin{tabular}{lcccc}
\hline & Group 1 & Group 2 & Group 3 & P Value \\
\hline Procedural time (minute) & $74.20 \pm 38.15$ & $68.50 \pm 36.63$ & $66.10 \pm 32.36$ & 0.516 \\
Systolic blood pressure (mmHg) & $126.00 \pm 27.77$ & $122.95 \pm 19.82$ & $127.50 \pm 21.18$ & 0.733 \\
Diastolic blood pressure (mmHg) & $78.64 \pm 15.15$ & $78.25 \pm 9.64$ & $77.96 \pm 11.62$ & 0.966 \\
Heparin $\left(\mathrm{IU} / 10^{3}\right.$ ) & $6.91 \pm 2.96$ & $8.40 \pm 3.40$ & $7.74 \pm 3.04$ & 0.150 \\
Interventional procedure & $24(48 \%)$ & $12(60 \%)$ & $35(70 \%)$ & 0.081 \\
Right radial access & $46(92 \%)$ & $18(90 \%)$ & $40(80 \%)$ & 0.188 \\
IIb/IIIa glycoprotein inhibitors & $3(6 \%)$ & $4(20 \%)$ & $7(14 \%)$ & 0.205 \\
Activated clotting time at the end of procedure (second) & $267.84 \pm 71.99$ & $266.10 \pm 63.69$ & $267.24 \pm 55.82$ & 0.995 \\
\hline
\end{tabular}




\section{SHORT-TIME RADIAL COMPRESSION WITH KAOLIN-FILLED PAD}

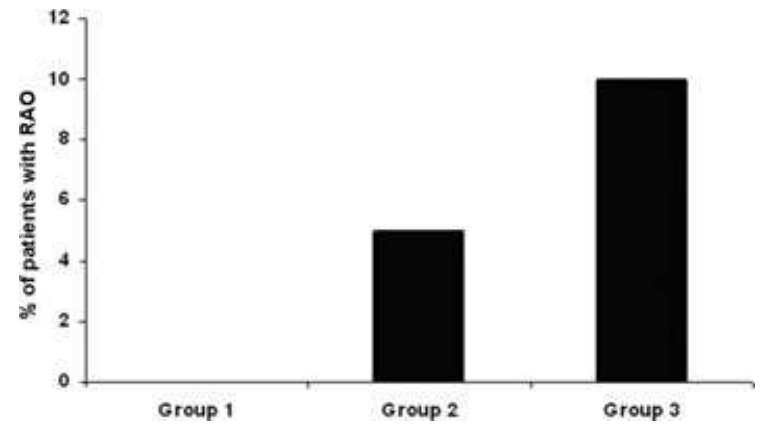

Figure 1. Incidence of radial artery occlusion at 24 hours $(\mathrm{P}=$ $0.05)$.

$20 \%$ of bleeding may seem high, it is worth noting that QuikClot ${ }^{\circledR}$ failure was associated with a chronic administration of double antiplatelet therapy and high levels of ACT after sheet removal. In particular at the univariate analysis, patients receiving clopidogrel were 28 times more likely to have early bleeding after compression removal, and ACT values greater than 287 seconds represented a strong predictor of bleeding with $80 \%$ sensitivity and $75 \%$ specificity. Nevertheless, in all patients who had QuikClot ${ }^{\circledR}$ failure, conventional compression was successfully performed with no further bleeding complications.

To date, there are no published studies reporting the use of QuikClot ${ }^{\circledR}$ Interventional ${ }^{\mathrm{TM}}$ hemostatic bandage after percutaneous coronary or peripheral procedures associated to a short-time compression to obtain early closure of radial access without bleeding complications.

Table 3. Univariate Predictors of Radial Artery Occlusion among Patients in Group 3

\begin{tabular}{lccc}
\hline & OR & $95 \%$ CI & P Value \\
\hline Age & 1.09 & $1.00-1.18$ & 0.049 \\
Male sex & 4.64 & $0.68-31.42$ & 0.116 \\
Weight & 1.11 & $0.99-1.24$ & 0.053 \\
Chronic clopidogrel therapy & 1.14 & $0.11-11.41$ & 0.909 \\
LMWH & 0.39 & $0.03-4.39$ & 0.446 \\
Interventional procedure & 4.12 & $0.61-27.78$ & 0.145 \\
Procedural time & 1.02 & $0.98-1.06$ & 0.338 \\
Systolic blood pressure & 0.99 & $0.95-1.03$ & 0.644 \\
IIb/IIIa glycoprotein inhibitor & 2.11 & $0.17-25.92$ & 0.599 \\
Heparin (IU/10 3 ) & 0.70 & $0.49-0.99$ & 0.044 \\
Activated clotting time & 0.99 & $0.97-1.01$ & 0.110 \\
\hline
\end{tabular}

Abbreviations as in Table 1.

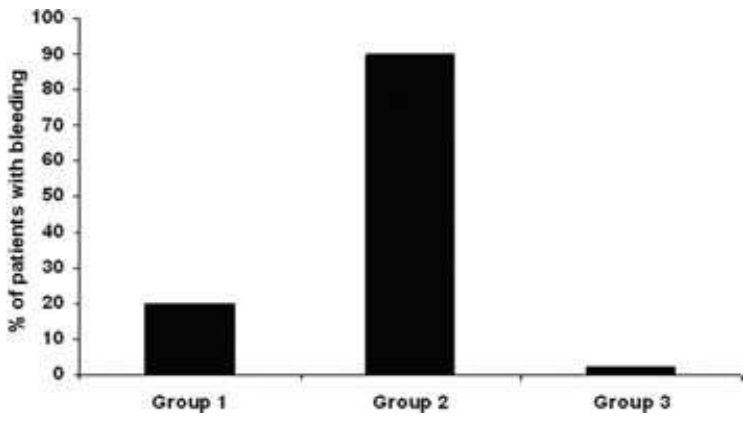

Figure 2. Incidence of active bleeding after compression removal $(\mathrm{P}<0.001)$.

The QuikClot system consists of a soft, white, sterile, hydrophilic nonwoven pad that should be applied topically together with a $3 \mathrm{M}^{\circledR}$ Tegaderm ${ }^{\mathrm{TM}}$ adhesive bandage. The QuikClot system has been used over the past 6 years by United States troops in the battlefield for treating external compressible hemorrhages. QuikClot favors arterial closure by two mechanisms. First, it acts as a selective sponge by absorbing water and concentrating coagulation factors at the site of wound. Second, the pad is filled with Kaolin, a clay mineral that activates the XII factor of coagulation accelerating the coagulation cascade (Fig. 4). QuikClot Interventional is a pad simply applicable at the site of puncture that does not require a learning curve for the operator or cath lab personnel or a patient monitoring after removing at the end of 15 minutes compression. This may be relevant in terms of costs reduction because the other currently available compression tools (e.g., TRband, Terumo Corporation, Japan) usually require a specific training for nurses or cath lab operators and a

Table 4. Univariate Predictors of Failure of Closure Technique among Group 1 Patients

\begin{tabular}{lccc}
\hline & OR & $95 \%$ CI & P Value \\
\hline Age & 0.99 & $0.94-1.06$ & 0.980 \\
Male sex & 1.52 & $0.28-8.28$ & 0.630 \\
BSA & 0.50 & $0.01-45.15$ & 0.760 \\
Ongoing clopidogrel & 28.78 & $4.79-172.82$ & $<0.001$ \\
LMWH & 0.52 & $0.57-4.83$ & 0.568 \\
Warfarin before hospital admission & 0.78 & $0.08-7.51$ & 0.828 \\
Interventional procedure & 6.00 & $1.12-31.98$ & 0.036 \\
Procedural time (minute) & 0.99 & $0.98-1.01$ & 0.911 \\
Systolic blood pressure & 1.00 & $0.98-1.03$ & 0.608 \\
IIb/IIIa glycoprotein inhibitors & 2.11 & $0.17-25.92$ & 0.599 \\
Heparin (IU/10 ${ }^{3}$ ) & 1.00 & $1.00-1.00$ & 0.155 \\
Activated clotting time (second) & 1.02 & $1.00-1.02$ & 0.009 \\
\hline
\end{tabular}


POLITI, ET AL.

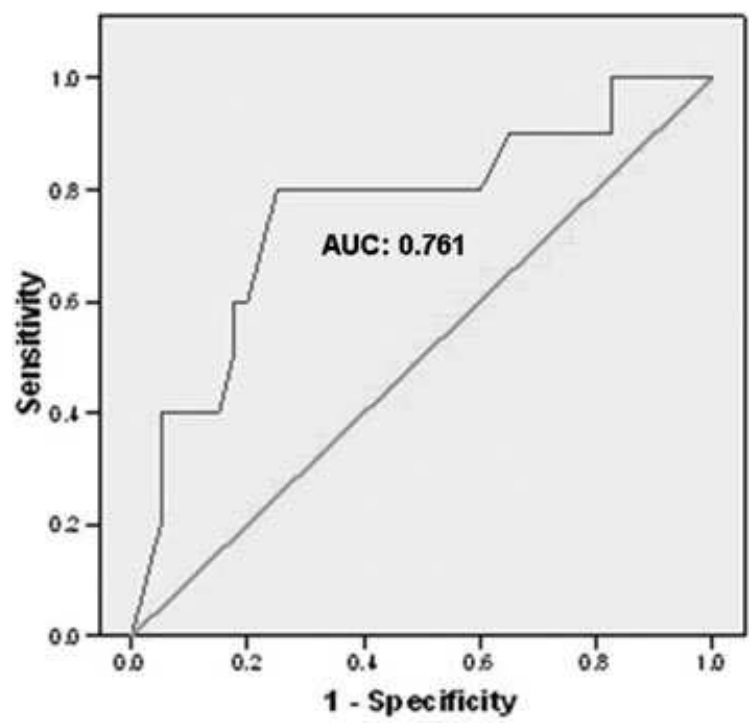

Figure 3. The blue line represents the ROC curve describing the sensibility and the specificity of ACT in predicting the device failure (active bleeding after 15 minutes removal) in group 1. As the area under the curve is 0.761 and the curve is on the left of neutral line (green), we can assume that ACT has a good accuracy in predicting the event. continuous monitoring for progressive compression decrease related to device deflation.

\section{Clinical Implications}

This study demonstrated that QuikClot ${ }^{\circledR}$ Interventional $^{\mathrm{TM}}$ is a useful option in the hands of interventional operators to obtain quick hemostasis of the radial artery with a reduced risk of RAO compared to conventional techniques. The present study suggests that the maximum benefit of this technique can be obtained especially in patients who are not on double antiplatelets therapy or who have lower values of ACT at the end of the procedure. Thus, waiting for lower ACT values before sheath removal is a reasonable option to reduce device failure.

\section{Strengths and Limitations}

To the best of our knowledge, this is the first randomized study to compare the QuikClot ${ }^{\mathrm{R}}$ Interventional $^{\mathrm{TM}}$

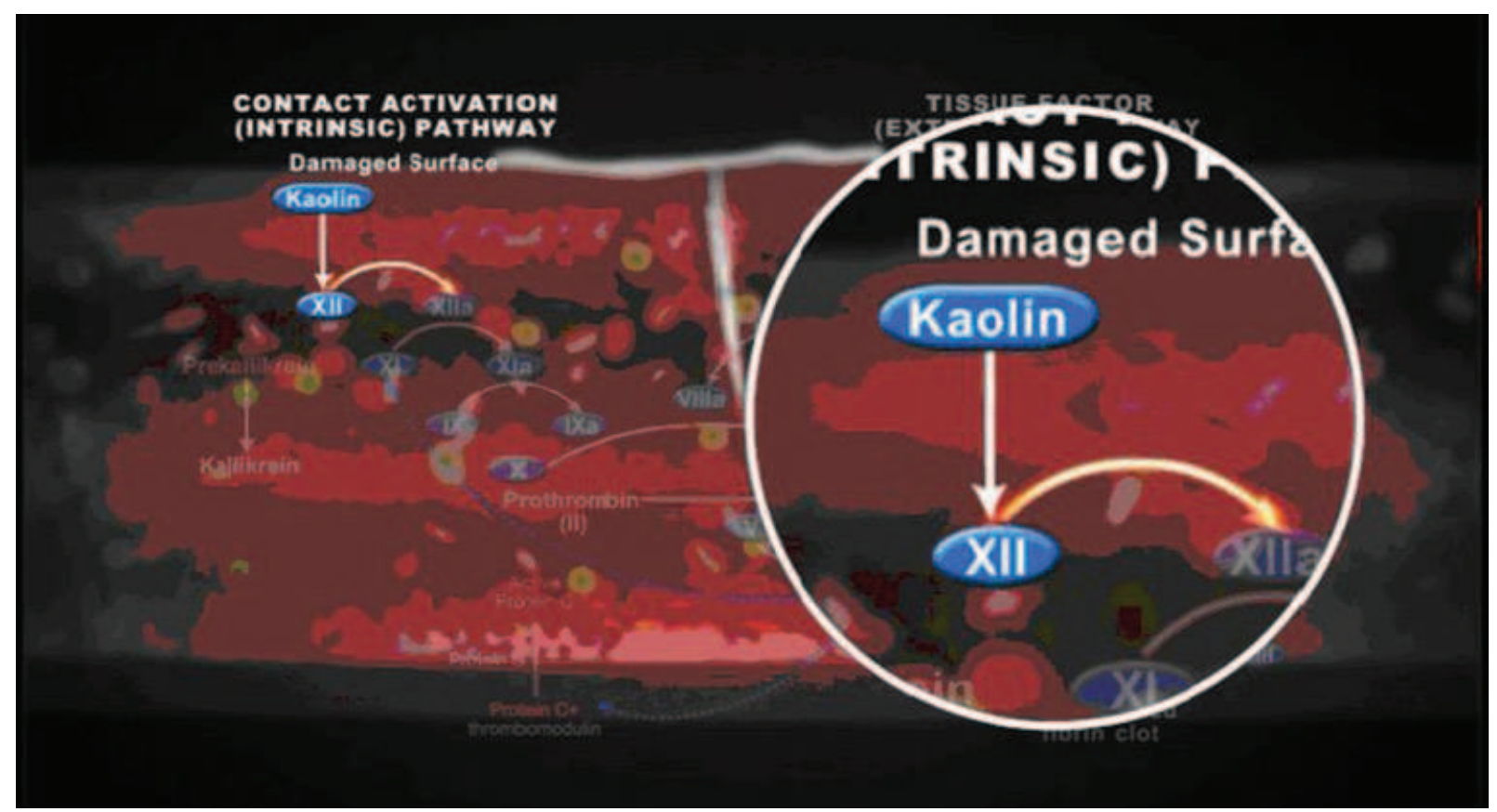

Figure 4. Role of Kaolin in coagulative cascade. 


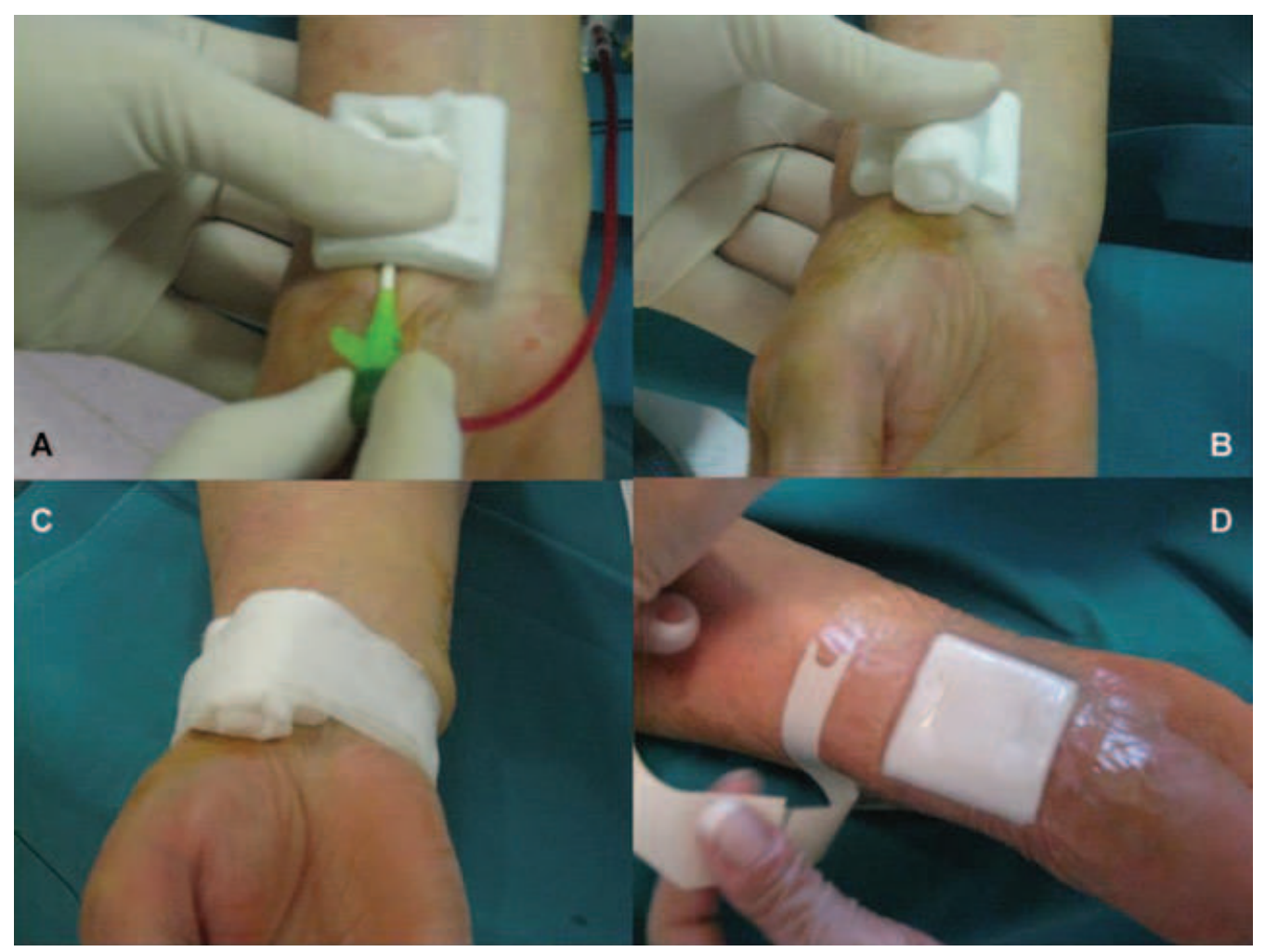

Figure 5. Panel A. QuikClot ${ }^{\circledR}$ Interventional ${ }^{\mathrm{TM}}$ hemostatic pad applied directly on the skin just before removing the radial sheath. Panel B. Initial brief manual compression with a folded gauze upon the pad. Panel C. Dressing wrapped with adhesive tape and maintained for 15 minutes. Panel D. The compression is removed leaving the QuikClot ${ }^{\circledR}$ Interventional ${ }^{\mathrm{TM}}$ secured with a Tegaderm ${ }^{\mathrm{TM}}$ adhesive bandage.

for radial access closure in comparison to conventional techniques in a series of consecutive patients undergoing coronary angiography or angioplasty. Because this was a trial testing a new device and a new technique, the number of participants was limited to that necessary to test the main study end-point. However, the limited number of exclusion criteria enhances the generalizability of the results. We have not assessed chronic RAO by Barbeau's test; therefore, it may be that some patients with RAO at 24 hours in group 3 would eventually recanalize at 30 days as previously shown. ${ }^{13}$ However, the advantage of QuikClot in group 1 remains evident as no patient developed RAO. Similarly to other small size trials with minor hazards, there was not an external independent safety monitoring board, but the study investigators monitored the study for the occurrence of adverse effects. Indeed, due to an unexpectedly high level of failure in one group, the study investigators decided to stop early one arm of the study for futility. Of note such complication was minor, and none of the patients enrolled in the trial developed major adverse events. Finally, there is an imbalance in interventional procedures with elective or bailout IIb/IIIaglycoprotein inhibitors-inhibitors administration among group 1 and 3 . This is the result of a blind randomization performed before coronary angiography that could have produced an underestimation of RAO incidence in group 3 for the higher antiplatelets regimen respect to group 1.

\section{Conclusions}

The present randomized clinical trial demonstrated for the first time that a novel hemostatic technique based on QuikClot ${ }^{\circledR}$ Interventional $^{\mathrm{TM}}$ pad utilization combined with short-time compression is significantly superior to conventional compression technique in reducing the risk of RAO after percutaneous transradial coronary procedures. Furthermore, the study shows 
POLITI, ET AL.

that early sheet removal and short-time compression with QuikClot ${ }^{\circledR}$ Interventional ${ }^{\mathrm{TM}}$ is safe and effective especially in patients not on double antiplatelet therapy and can be performed routinely after diagnostic or interventional procedures. Despite QuikClot ${ }^{\circledR}$ Interventional $^{\mathrm{TM}}$ application, early sheet removal with an ACT higher than 287 seconds or in patients taking chronic dual antiplatelet therapy should be cautious due to the risk of hemorrhage at the puncture site.

\section{References}

1. Mann T, Cowper PA, Peterson ED, et al. Transradial coronary stenting: Comparison with femoral access closed with an arterial suture device. Catheter Cardiovasc Interv 2000;49(2):150 156.

2. Sciahbasi A, Fischetti D, Picciolo A, et al. Transradial access compared with femoral puncture closure devices in percutaneous coronary procedures. Int J Cardiol 2009;137(3):199_ 205.

3. Pristipino C, Trani C, Nazzaro MS, et al. Major improvement of percutaneous cardiovascular procedure outcomes with radial artery catheterisation: Results from the PREVAIL study. Heart 2009;95(6):476-482.

4. Sanmartin M, Gomez M, Rumoroso JR, et al. Interruption of blood flow during compression and radial artery occlusion after transradial catheterization. Catheter Cardiovasc Interv 2007;70(2):185-189.
5. Chatelain P, Arceo A, Rombaut E, et al. New device for compression of the radial artery after diagnostic and interventional cardiac procedures. Cathet Cardiovasc Diagn 1997;40(3):297300 .

6. Stella PR, Kiemeneij F, Laarman GJ, et al. Incidence and outcome of radial artery occlusion following transradial artery coronary angioplasty. Cathet Cardiovasc Diagn 1997;40(2):156-158.

7. Nagai S, Abe S, Sato T, et al. Ultrasonic assessment of vascular complications in coronary angiography and angioplasty after transradial approach. Am J Cardiol 1999;83(2):180-186.

8. Ochiai M, Sakai H, Takeshita S, et al. Efficacy of a new hemostatic device, Adapty, after transradial coronary angiography and intervention. J Invasive Cardiol 2000;12(12):618622.

9. Choi EY, Ko YG, Kim JB, et al. Hemostatic efficacy of hydrophilic wound dressing after transradial catheterization. J Invasive Cardiol 2005;17(9):459-462.

10. Cox ED, Schreiber MA, McManus J, et al. New hemostatic agents in the combat setting. Transfusion 2009;49(Suppl 5): $248 \mathrm{~S}-255 \mathrm{~S}$

11. Arnaud F, Teranishi K, Tomori T, et al. Comparison of 10 hemostatic dressings in a groin puncture model in swine. J Vasc Surg 2009;50(3):632-639, e631.

12. Barbeau GR, Arsenault F, Dugas L, et al. Evaluation of the ulnopalmar arterial arches with pulse oximetry and plethysmography: Comparison with the Allen's test in 1010 patients. Am Heart J 2004;147(3):489-493.

13. Pancholy SB. Comparison of the effect of intra-arterial versus intravenous heparin on radial artery occlusion after transradial catheterization. Am J Cardiol 2009;104(8):10831085. 